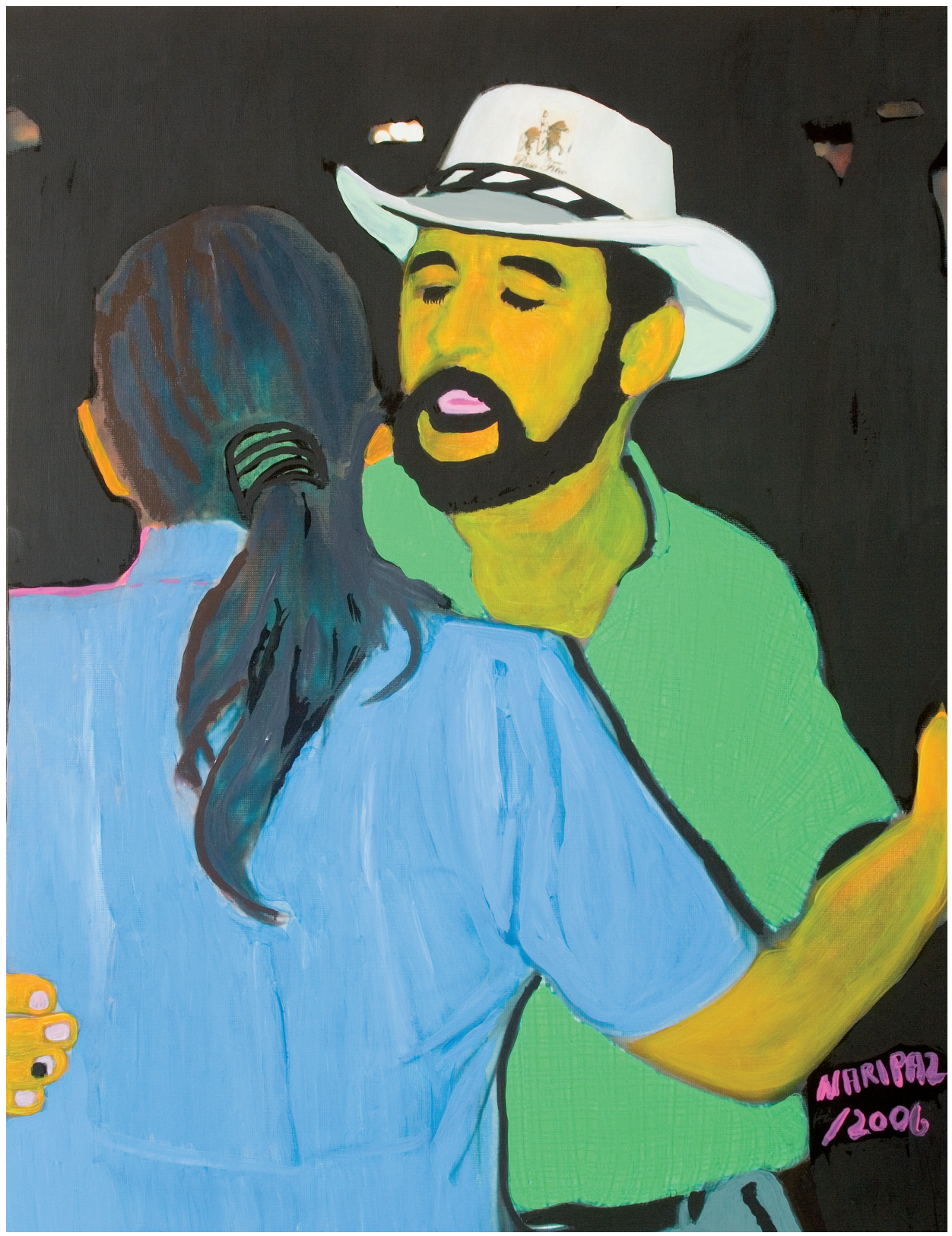

Serie Bailando por un sueño. Bolero

Acrílico / tela. 2006. 72 x 55,5 cms.

Maripaz Jaramillo 


\section{Dualidad: competencias genéricas- dispositivos pedagógicos como estrategia para el aprendizaje en educación virtual'}

Fecha de recepción: 2 de noviembre de 2010 - Aceptación: 16 de diciembre de 2010

\section{María del Socorro GuZmán Serna}

\section{Resumen}

Este artículo, hace parte del proyecto "Diseño del modelo para la evaluación de las competencias: comunicativa, tecnológica e investigativa, en la modalidad de Educación Virtual de la Institución Universitaria Politécnico Grancolombiano", en el cual se muestran algunos hallazgos que dan cuenta del papel dual que juegan las Competencias comunicativa, tecnológica e investigativa, denominadas Competencias genéricas, en la estructura de la propuesta virtual del Politécnico. Por una parte, el modelo virtual busca que estas competencias sean un aporte en la formación profesional y en la vida de los estudiantes y por otra, muestra cómo las mismas son dispositivos pedagógicos que le permiten a la propuesta virtual del Politécnico realizar su gestión y apropiación de conocimiento.

El contenido del trabajo se dividirá en tres partes: en primera instancia, se precisarán algunos conceptos básicos para clarificar los temas abordados; en segundo lugar, se presentará la experiencia del Politécnico con las competencias y su papel dual como estrategia para el aprendizaje en Educación Virtual y, por último, se presentarán algunas conclusiones.

\section{Abstract}

This article is part of the project "Design of the Model for Evaluating the Communicative, Technological, and Investigative Competences in the Virtual Education
Modality of the Politécnico Grancolombiano University Institution". This project shows some findings that describe the dual role of the Communicative, Technological, and Investigative Competences, which are called Generic Competences, within the structure of the virtual education proposal of Politécnico. On one hand, the virtual model seeks for these competences to contribute to the professional training and student lives. On the other hand, it shows how these competences are used as educational devices that allow the virtual education proposal of Politécnico to be accurate and efficient.

The project is divided in three parts: First, some basic concepts will be explained in order to clarify the topics seen; second, the experience at Politécnico, as well as the competences and their dual role as the strategy for a correct Virtual Education learning, will be presented; and lastly, some conclusions will be drawn.

\section{Palabras clave}

Competencias genéricas, dispositivo pedagógico, prácticas pedagógicas, educación virtual.

\section{Keywords}

Generic Competitions, educational device, educational practices, virtual education.

1. El proyecto de investigación del cual es producto este documento ha sido financiado por la Fundación Politécnico Grancolombiano Institución Universitaria mediante el contrato de investigación 2010-DI-EI-BC-3, del 14 de diciembre de 2009. 
El enfoque de competencias en el ámbito educativo, desde la perspectiva del Ministerio de Educación en Colombia (MEN), ha sido asumido como parte de las estrategias para garantizar la calidad académicay la articulación con los diferentes niveles formativos.

\section{Introducción}

La tendencia de la Educación Superior en estas últimas décadas se ha encaminado a promover $y$ fomentar su proceso de enseñanza-aprendizaje o aprendizaje por competencias, con el propósito de ir acercando el mundo educativo al mundo productivo. La perspectiva de este acercamiento pretende responder a la optimización de la calidad, a la formación permanente, a la competitividad profesional y laboral.

Estos propósitos han llevado a la Educación Superior a replantearse la concepción de formación y a profundizar en el concepto y manejo de una formación integral en donde el ser humano debe ser asumido como una unidad integrada por lo físico, lo biológico, lo emocional, lo social, lo cultural y lo histórico. Asumida así la formación integral, la Educación Superior tiene como reto integrar el currículo con la realidad. Este desafío se hace mayor en la Educación Virtual ya que conlleva diseñar y gestionar un currículo en donde su Propuesta Pedagógica dé cuenta de la integración con el contexto en un mundo cada día más cambiante.

Desde otra perspectiva, avanzar en los propósitos de mejoramiento de la calidad académica en la Educación Virtual implica

\section{Reseña de autor \\ María del Socorro Guzmán Serna (Colombia) \\ Politécnico Grancolombiano \\ mdsguzman@poligran.edu.co}

Licenciada en Literatura y Lengua Española, Universidad del Cauca, especialista en Ambientes Virtuales de Aprendizaje (OEA-Virtual Educa), experta en Administración de Negocios Internacionales, EAN y magíster en Educación de la Universidad Pedagógica Nacional. Integrante de los Grupos de investigación Gestión Vital y Educación. Cuenta con libros y artículos publicados. Actualmente, es la Directora Académica de Educación Virtual del Politécnico Grancolombiano. incorporar la función investigativa dentro de los sistemas de gestión de aprendizaje, por ello, este trabajo tiene como objetivo visibilizar la experiencia del Politécnico en su práctica pedagógica con el manejo de la dualidad: Competencias genéricas-Dispositivos pedagógicos como estrategias de aprendizaje en Educación Virtual.

\section{Algunos conceptos básicos \\ Las Competencias genéricas}

Se definen como aquellas que son comunes a la gran mayoría de las profesiones y se relacionan con conocimientos, valores, rasgos de personalidad, aptitudes, aspectos requeridos por varias ocupaciones laborales o transferibles en actividades, sectores u organizaciones.

El enfoque de competencias en el ámbito educativo, desde la perspectiva del Ministerio de Educación en Colombia (MEN), ha sido asumido como parte de las estrategias para garantizar la calidad académica y la articulación con los diferentes niveles formativos. Es de resaltar que desde el año 2008, el MEN definió como Competencias genéricas para la Educación Superior las siguientes: Comunicación en lengua materna y otra lengua internacional; Pensamiento Matemático; Ciudadanía y Ciencia; Tecnología y Manejo de la Información con las cuales pretende responder a:

"las necesidades del mundo globalizado, en el que las distancias se han acortado con los medios de comunicación y de transporte, las fronteras económicas y laborales tienden a difuminarse y la educación busca cada vez con más fuerza la internacionalización de los saberes, las profesiones, las titulaciones y los mercados 
de trabajo. Sin descuidar, por supuesto, la pertinencia frente al contexto local, para el cual estas competencias también constituyen una fuente de desarrollo, de modo que su implementación contribuirá al mejoramiento de la calidad de la formación y a su proyección internacional.

La pertinencia y la calidad de la Educación Superior dependen en gran medida de la implementación de cuatro competencias genéricas que permiten aprender durante toda la vida, formando profesionales capaces de moverse con destreza en el mundo globalizado".

El trabajo parte de estos preceptos y muestra los hallazgos en la propuesta del Politécnico.

\section{Dispositivo pedagógico}

En este caso se retoma la postura de Bernstein (1998) el cual asume el dispositivo pedagógico como aquel que:

"Proporciona la gramática intrínseca del discurso pedagógico (es decir, gramática, en sentido metafórico)...Regula fundamentalmente la comunicación que hace posible y, así, actúa de forma selectiva sobre el potencial del significado. El dispositivo regula continuamente el universo ideal de significados pedagógicos potenciales, restringiendo o reforzando sus realizaciones, (...) El dispositivo pedagógico hace posible un gran conjunto potencial de resultados comunicativos, (...) las formas de realización del dispositivo pedagógico están sujetas a reglas que varían con el contexto,(...) las reglas intrínsecas del dispositivo no están libres de ideología...las reglas participan esencialmente en la distribución de las diversas formas de conciencia y en las limitaciones que se le imponen... El dispositivo se convierte en lugar de apropiación, conflicto y control... Con el dispositivo pedagógico es posible obtener un resultado, una forma de comunicación que puede subvertir las reglas fundamentales del dispositivo".
Asimismo, plantea que "la naturaleza de un dispositivo pedagógico consta de tres conjuntos de reglas: distributivas, de recontextualizaciòn y evaluativas, y que esas reglas están en relación jerárquica unas respecto de otras... La regla uno distribuye conciencia a diferentes grupos, la regla dos regula la Constitución del discurso pedagógico específico y la regla tres constituye la práctica pedagógica”. (Bernstein, 1998).

Por otra parte, destaca este autor que el dispositivo pedagógico:

“es el medio a través del cual el poder puede ser relacionado con el conocimiento y el conocimiento con la conciencia". (Bernstein) y resalta, además, que "Un dispositivo pedagógico hace posible lo imaginario y por eso crea un lugar para lo ideológico".

\section{Experiencia en educación virtual}

La propuesta de Educación Virtual del Politécnico Grancolombiano recoge todas las políticas, preceptos, lineamientos y prácticas de la Institución y desde su plan de mejoramiento continuo viene reflexionando, experimentando y consolidando, para los programas de Educación Virtual, el manejo de tres competencias genéricas: la comunicativa, la investigativa y la tecnológica, competencias que a su vez se han convertido en dispositivos pedagógicos como estrategias para el aprendizaje.

Dado que la apuesta educativa del Politécnico privilegia el aprendizaje autónomo y significativo y centra dichos procesos en el estudiante y que la estructura del modelo virtual permite utilizar diferentes ambientes de aprendizaje para flexibilizar el currículo, conviene dinamizar a través de los materiales didácticos, propuestas de proyectos cercanos a la realidad de los estu- 
diantes, hacer uso de la creatividad didáctica para generar comunicación e interacción permanente a través de diferentes recursos y medios y hacer uso permanente de la tecnología como medio y como herramienta. El área de Educación Virtual diseñó y viene desarrollando seguimiento a estos tres ejes: comunicativos, investigativos y tecnológicos (que además son política y eje estructural de la Institución) como agentes que permiten la dualidad: Competencias genéricas-Dispositivos pedagógicos, con el propósito de dar cuenta de la formación integral por Competencias genéricas y la autonomía intelectual que la Institución propone.

Para el diseño y desarrollo de esta estructura se asumieron, además de los marcos de referencia y preceptos establecidos por la Institución, el aprendizaje autónomo, la formación integral por competencias, las posibilidades que permite la modalidad respecto al manejo del espacio-tiempo, la calidad académica, el acompañamiento permanente de tutores a través de las TIC y las dinámicas de los espacios de aprendizaje, así como criterios para su evaluación.

El marco anterior permitió estructurar la propuesta a partir de cuatro pilares fundamentales: los Ejes Institucionales para Educación Virtual, los Ambientes y Momentos de Aprendizaje, el Aprendizaje Autónomo y el Autoaprendizaje. Apoyos a través de los cuales se gestionan todos los procesos del aprendizaje que a continuación se desglosan.

\section{Ejes Institucionales para la Educación Virtual}

Modalidad virtual: demanda programas diseñados y desarrollados para el aprendizaje personalizado, participativo, autó- nomo, flexible, con comunicación bidireccional y mediatizada por recursos didácticos.

Modelo por competencias: incluye las genéricas, básicas, de pensamiento, profesionales y socio-humanísticas a partir de los preceptos institucionales de saber ser, saber hacer, saber aprender y saber emprender.

Cadenas de formación: ciclos propedéuticos e interacción entre la educación formal y continuada.

Formación dual: interrelación entre teoría y práctica; entre conocimiento y contexto, y entre unidad e integración (formación investigativa - proyección social).

\section{Ambientes y momentos de aprendizaje}

Está conformado por tres momentos para el aprendizaje:

- Teleconferencia teórica y práctica

- Trabajo colaborativo

- Trabajo autónomo y acompañado a través del aula virtual

\section{Teleconferencia}

Contempla los siguientes objetivos:

- contextualizar e introducir las temáticas

- reforzar el aprendizaje autónomo

- presentar experiencias prácticas o vivencias de aplicación del conocimiento en la vida real

- contribuir al desarrollo de las competencias del saber aprender y saber hacer a través de las didácticas utilizadas por el expositor y retroalimentar resultados del aprendizaje.

\section{Trabajo colaborativo}

Es un espacio de encuentro presencial o virtual entre los estudiantes y de ellos, con los tutores de manera virtual. Está orientado 
a la ejecución de actividades de grupo que contribuyan al desarrollo de las competencias, específicamente las relacionadas con el trabajo en equipo, control emocional, manejo de conflictos, solución de problemas y manejo efectivo del tiempo. Tiene como objetivos: reforzar el aprendizaje autónomo; desarrollar la actividad de trabajo colaborativo; contribuir al desarrollo de las competencias del saber aprender y saber hacer a través de las didácticas planteadas.

- Reforzar las Competencias sociales para trabajar en equipo.

- Realizar evaluaciones programadas por el autor.

- Aplicar metodologías para equipos de alto rendimiento que faciliten, refuercen y enriquezcan el proceso de aprendizaje individual y colectivo.

\section{Aula Virtual}

Espacio diseñado para cumplir con la función académica de comunicación e interacción entre estudiante - tutor, estudiante estudiante, estudiante - grupo académico. Contiene los módulos, materiales, enlaces, vínculos y espacios como foro general, foro temático, chat, mensajería y mesa de ayuda.

\section{Aprendizaje autónomo}

Corresponde a los momentos de trabajo académico que hace el estudiante, los cuales deben ser programados con base en los objetivos de aprendizaje/ competencias que espera alcanzar en el módulo y apunta a una capacitación en:

- indagar, contrastar fuentes, tomar decisiones y argumentar

- resolver preguntas, diagnosticar y proponer soluciones
- aplicar teorías a objetos concretos en contextos diferentes

- proponer nuevas soluciones a procesos y procedimientos en contextos diferentes

- tener una disciplina metodológica que le permita indagar, evaluar, aplicar y ordenar la importancia de los resultados.

\section{Auto aprendizaje}

Implica, de parte del estudiante asumir:

- el seguimiento a los contenidos académicos correspondientes a la disciplina de estudio del material didáctico asignado por la Institución, la indagación de fuentes bibliográficas, la consulta de material de apoyo y la participación activa en los foros, chat y proyecto que convoque su tutor.

- los espacios para la formación investigativa: dados a partir de proyectos de autoestudio, proyectos de aula, semilleros de investigación y células investigativas.

De esta manera se ha venido dinamizando, gestionando y articulando el proceso de aprendizaje por competencias, el manejo espacio-tiempo, las dinámicas de aprendizaje, el acompañamiento del tutor y sus interacciones. Esta estructura ha permitido gestionar las competencias tanto comunicativa, investigativa y tecnológica de la siguiente manera:

\section{La Competencia comunicativa}

Considerada en el Politécnico una de las competencias de mayor trascendencia bien en el ámbito académico como en el ámbito laboral por ser parte fundamental del ser humano. Por ello, en su estructura viene orientando un proceso lecto-escritor direccionado a la argumentación, la sustentación y la comprensión. Con este propósito se han estructurado las siguientes 
Estrategias Pedagógicas:

\section{Ambientes de aprendizaje}

Teleconferencias en vivo, aula virtual, particularmente a través de los recursos comunicativos que le brinda la plataforma; foro, chat, mensajería, espacios donde permanentemente ponen a prueba sus habilidades escritas, lectoras y orales.

\section{Diseño de actividades}

En la estructura de los módulos se cuenta con estrategias para que los estudiantes lean, escuchen, vean y compartan diferentes perspectivas con sus compañeros para enfrentar la realidad o una experiencia determinada; compartir observaciones, identificar y aceptar diferencias, participar en generación de preguntas, comunicar sus observaciones, compartir y proponer ideas, formular y reformular posibles explicaciones a través del debate, la discusión, acordar y planificar estrategias de comunicación, crear y comprender textos escritos, con requerimientos técnicos en la escritura, la comunicación y el significado de los símbolos escritos.

\section{Módulo}

El plan de estudios incluyó el módulo Técnicas del Aprendizaje Autónomo, que busca apoyar y contextualizar con los requerimientos de hábitos de estudio propios de la modalidad, los procesos de aprendizaje autónomo y los procesos lectoescritores.

Por otra parte, la estructura de los módulos al incluir audiovisuales, mapas, gráficas, fotografías, OVAS, audios como interacción educativa, le permite al estudiante producir, receptar e interpretar mensajes de diferentes tipos.

La expresión, la capacidad de escucha y la interpretación son fundamentales para hacerse comprender y entenderse con los otros; esta complejidad requiere y abarca una serie de actividades, tareas, conocimientos, habilidades, actitudes y valores que permiten realizar funciones específicas.

Contribuir específicamente con el desarrollo de actitudes y habilidades en la modalidad virtual requiere sobrepasar las limitaciones de espacio-tiempo para centrar la atención en cada contexto específico. En este proceso, el acercamiento, la comunicación y la participación son vitales para plantear necesidades, acuerdos y desacuerdos, entre otros. Es parte de la estrategia que se den encuentros entre actores (tutores-estudiantes, estudiantes-estudiantes) por vías como las teleconferencias en vivo, los chat, la mensajería, el correo y los foros, en donde el tutor juega un papel fundamental en la invitación, motivación y articulación grupal, el incentivo permanente a la participación, aspectos que permiten que se dé la comunicación e interacción de manera permanente, eficaz, fluida y en forma multidireccional.

\section{Relación con la competencia ciudadana (valores)}

El lenguaje permite a los diferentes actores ponerse de acuerdo. En Educación Virtual no se requiere de la presencia física para referirse a algo, independiente de si es tangible o intangible. Se cree en los otros, creencia que se fundamenta en la capacidad que tienen las personas de expresar sus vivencias pasadas y presentes, además de pensar y planear el futuro. El lenguaje ha llevado a estar en constante construcción de conocimientos en diferentes tipos de áreas, lo que se denomina Cultura del conocimiento La Competencia investigativa.

Esta requiere, para su proceso de apropiación, de la capacidad de usar el lenguaje 
hablado y escrito, de la reflexión crítica sobre la información en sí misma, es decir, el manejo de estructuras, cultura, contexto e impacto.

El manejo de la información en la investigación es base fundamental para entender alternativas, opciones, formación de opiniones y toma de decisiones basadas en la información de manera responsable. El manejo de la información debe permitir:

- reconocer lo conocido y determinar lo desconocido

- identificar, ubicar y acceder a fuentes de información de manera adecuada (incluida la red)

- evaluar la calidad y pertinencia de la información

- organizar la información y el conocimiento

Pensar y actuar requiere reflexionar desde la capacidad de enfrentar cambios, aprender de la experiencia, pensar y actuar con una postura crítica, además de aplicar fórmulas y métodos para enfrentar una situación.

Desde esta perspectiva, la estructura de investigación formativa se acciona desde tres procesos pedagógicos: el primero lo realizan a través del proceso de Aprendizaje autónomo, el cual está enfocado a que el estudiante realice búsquedas de fuentes, haga exploraciones, describa y correlacione con la realidad preguntas, problemáticas y supuestos; El segundo proceso lo debe realizar en cada módulo a través del Proyecto de Aula o Autoestudio; y el tercer proceso lo lleva a cabo a través del Proyecto de Área. Este proyecto de área se desarrolla de manera continua articulando 3 o 4 módulos del bloque de programa. El proyecto debe dar cuenta de los siguientes aspectos:
Las TIC tienen el potencial para transformarla manera de trabajar juntos, acceder a la información e interactuar con los demás y para ello es necesario relacionar posibilidades, herramientas $y$ metas.

Proyecto de Investigación

\begin{tabular}{|c|c|c|c|c|c|c|}
\hline \multirow[t]{2}{*}{ Programa } & Módulo & \multicolumn{2}{|c|}{$\begin{array}{l}\text { Tipo de } \\
\text { Investigación }\end{array}$} & $\begin{array}{l}\text { Ciclo } \\
\text { Propedéutico }\end{array}$ & Competencias & $\begin{array}{l}\text { Tema de } \\
\text { Investigación }\end{array}$ \\
\hline & $\begin{array}{l}1 . \\
2 . \\
3 .\end{array}$ & $\begin{array}{l}\text { Exploratc } \\
\text { Descripti } \\
\text { Correlaci }\end{array}$ & & & & \\
\hline $\begin{array}{l}\text { Línea de } \\
\text { Investigaci }\end{array}$ & $\begin{array}{l}\text { Dep } \\
\text { Aca }\end{array}$ & $\begin{array}{l}\text { rtamento } \\
\text { émico }\end{array}$ & Tutor & $\begin{array}{l}\text { Producto de } \\
\text { Entrega }\end{array}$ & $\begin{array}{l}\text { Investigación } \\
\text { Aplicada }\end{array}$ & Observaciones \\
\hline
\end{tabular}

A través de la estructura de proyecto se propone que el desempeño investigativo se desarrolle mediante un conjunto de experiencias

"que permitan procesos que van desde la ruptura con la postura tradicional frente a la existencia, hasta la elaboración de una estrategia argumentativa, pasando por el dominio de recursos diversos para refinar las preguntas, apropiarse y servirse de conceptos elaborados por otros, gracias a una lectura crítica que establece relaciones intra y extratextuales, realizar proceso de inves- tigación formativa que permitan vincular los propios interrogantes con pesquisas y preguntas formuladas por otros, reunir corpus pertinentes, tratar adecuadamente el material bibliográfico y documental, recoger y tratar en la debida forma toda suerte de datos primarios, establecer relaciones de causalidad, formular supuestos, hipótesis, someter la actuación a un derrotero metódico acorde con ellas, y servirse de la escritura como una herramienta de construcción del pensamiento, de elaboración de conceptos y de desarrollo argumentativo." (Camacho, 2007). 


\section{Relación con valores.}

En este sentido, se enfatiza y se trabaja con el estudiante desde la perspectiva del manejo y respeto por los derechos de autor, el respeto por las ideas y posturas de los demás, el reconocimiento y consenso compartido.

\section{La Competencia tecnológica.}

Hoy, el uso de la comunicación e interacción de manera eficaz con el medio, más que un requerimiento es una necesidad en la acción diaria. Se solicita que las personas puedan utilizar una gama amplia de las Tecnologías de la Comunicación e Información (TIC) y a su vez que manejen los diferentes lenguajes audiovisuales. Las relaciones e interacciones con otros, en grupos o redes, requieren del uso y manejo de herramientas interactivas para actuar de forma autónoma y con capacidad de asumir la responsabilidad de gestionar la vida y situarla en un contexto social más amplio, para reflexionar haciendo uso de habilidades metacognitivas (pensar sobre el pensamiento), habilidades creativas, postura crítica y para pensar en cómo construir, experimentar incluyendo pensamiento, sentimiento y relaciones sociales.

Lo anterior implica pensar y actuar de manera integrada, realizando interconexiones, interrelaciones múltiples entre posiciones e ideas que puedan parecer contradictorias o superficiales. Para ello, las herramientas tecnológicas deben ser Interactivas.

\section{¿Por qué se requiere el uso de herramientas Interactivas?}

Las demandas sociales, la economía y la sociedad de la información requieren de profesionales que dominen herramientas que les permitan interactuar con el conocimiento, la información y la comunicación, así como con el manejo de equipos.

\section{¿Por qué se requiere utilizar la tecnología de forma interactiva?}

Los avances tecnológicos han impuesto exigencias dentro y fuera del ámbito laboral, se requiere satisfacer con eficacia las demandas.

El uso de la tecnología requiere y exige de parte de los individuos la adopción de nuevas formas de conocimiento y uso en la vida cotidiana. Las TIC tienen el potencial para transformar la manera de trabajar juntos, acceder a la información e interactuar con los demás y para ello es necesario relacionar posibilidades, herramientas y metas.

¿Para qué? para estar a la vanguardia con las tecnologías; para responder a las necesidades personales, profesionales y laborales manteniendo así un diálogo activo con el mundo.

¿Cómo se logra? Teniendo acceso a la herramienta y conocimiento técnico para el manejo. Crear y adaptar requieren familiaridad con la herramienta, comprensión de cómo se dan los cambios y cómo pueden ser utilizados para otros objetivos. La herramienta debe convertirse en un instrumento activo entre el individuo y su medio.

La Competencia tecnológica en Educación Virtual inicia con el requerimiento que la misma modalidad exige para su proceso, por ello el plan de estudios incluyó el módulo "Herramientas para la productividad", el cual contempla el manejo del computador, el manejo del campus y aula virtual, así como el manejo del paquete de Office que son aspectos básicos que introducen a los estudiantes en la competencia. Posteriormente, con el uso y manejo del aula, actividades, herramientas, 
técnicas y estrategias, se aporta al desarrollo de habilidades para entender y emplear los lenguajes audiovisuales, los conocimientos, información, símbolos y formas interactivas.

En conjunto, se busca que los estudiantes logren el manejo o dominio técnico de software y hardware de cada uno de los medios que utilizan, que desarrollen habilidades para buscar, seleccionar, analizar y reflexionar sobre la información a la que tienen acceso a través del campus y video-conferencias, además que hagan uso de los medios como sistemas de expresión y comunicación. De esta manera se espera aportar a la percepción y a la forma como los estudiantes puedan relacionarse con el mundo.

Es de resaltar que las tres competencias también le aportan al estudiante en:

- La relación con los demás ya que le permite mantener y administrar las relaciones personales y de esta manera hace uso del manejo de la inteligencia emocional.

- La capacidad para respetar y apreciar valores, creencias, culturas e historias la cual se logra mediante el manejo de la empatía (ponerse en el lugar del otro e imaginar la situación desde su perspectiva).

- La gestión de las emociones es ser conscientes y capaces de interpretar los estados emocionales frente a otros.

- La capacidad de cooperar, competencia que requiere la habilidad de equilibrar compromisos con el grupo y los objetivos acorde con las prioridades.

- La capacidad de compartir el liderazgo y apoyar a otros para lo cual se requiere que presente ideas y escuche a los demás; que comprenda la dinámica (método) del debate y maneje su agenda; que establezca alianzas duraderas y sostenibles; que nego- cie y tome decisiones incluyendo la opinión de la gran mayoría.

- La capacidad para actuar en coherencia y consecuencia con lo expuesto, llevando a cabo planes de vida personal y proyectos y hacer valer derechos, límites y necesidades.

Asimismo, es importante destacar que del seguimiento y las reflexiones que viene realizando el área de Educación Virtual se dedujo que las tres competencias no se circunscriben a un momento y nivel, se dan durante todo el proceso académico que realiza el estudiante. Por ello se puede decir que se convierten en Competencias transversales. Los hallazgos también permiten indicar que dan cuenta de la política y ejes estructurales que la Institución resalta tanto en su misión como en su Proyecto Educativo Institucional respecto a promover la cultura de la investigación, impulsar el desarrollo informático y telemático, involucrar la ciencia y la tecnología, además de fomentar las Competencias lectoescritoras en todos sus planes de estudio.

Desde esta perspectiva, podemos decir que estas tres competencias son válidas en todas las profesiones, responden a las exigencias del mundo actual, son pertinentes en diversos contextos, contribuyen al mejoramiento de la calidad, la formación y la proyección internacional y permiten el aprendizaje para la vida. Por ello, podemos afirmar que son Competencias genéricas.

Estas tres competencias en virtual, además, son asumidas y elegidas con el criterio de Competencias capacitadoras en razón a que son competencias base o soportes fundamentales para desarrollar las demás competencias. Por lo tanto, com- 
petencias que se aprenden, apropian y se manejan toda la vida. De la misma manera, se resalta la presencia de las competencias ciudadanas en cada una de ellas. Por otra parte, cabe destacar que si bien se identifican por cada competencia los aspectos fundamentales que se deben apropiar y usar, en la práctica al tiempo se requiere el manejo de varias habilidades.
Desde el marco de los dispositivos, se propuso asumir el modelo pedagógico, comunicativo e instruccional de tercera generación como un gran eje transversal para gestionar el currículo. Este Eje Transversal se dinamiza con los dispositivos pedagógicos: comunicativo, tecnológico y el investigativo.

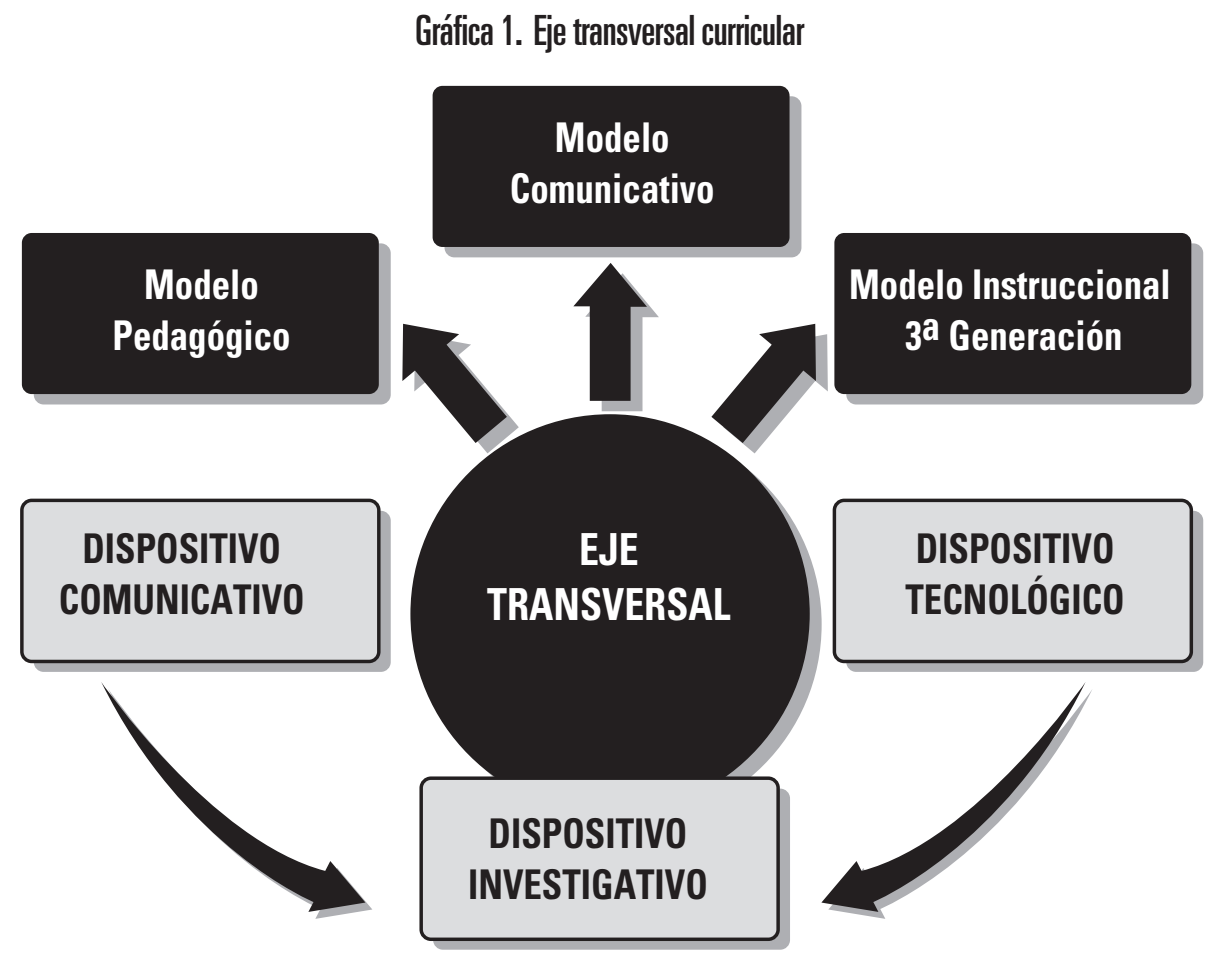

Desde esta perspectiva, el currículo se planea y gestiona a partir de tres escenarios: el aula virtual, el proyecto de investigación formativa (desde la perspectiva de confrontación teoría-práctica) y la práctica aplicada. En cada uno de estos contextos la comunicación, la investigación y la tecnología juegan los roles de competencias y de dispositivos. 


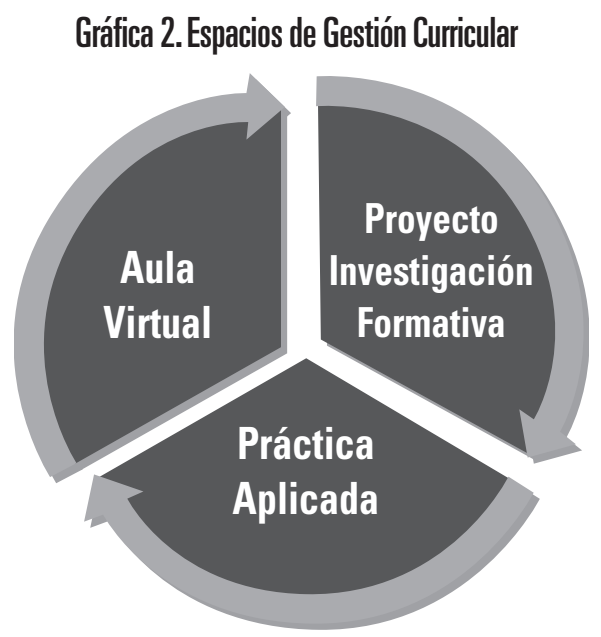

El Aula Virtual pasa a ser un espacio para la gestión del conocimiento dinamizado por ambientes de aprendizaje, que conllevan al intercambio de experiencias, la aplicación y experimentación del aprendizaje de manera autónoma, en donde encuentran distribuida la información de manera confiable y se evalúan los procesos. A su vez, permite a los actores: tutor-estudiantes, estudiantes-estudiantes, la facilidad de acceso, actualización constante, monitoreo, contar con archivos, vínculos, enlaces y en general materiales disponibles.

El Proyecto de Investigación Formativa se asume como confrontación teoría-práctica, tiene como propósito dinamizar y gestionar la construcción de conocimiento de manera colaborativa, en donde además de apropiar la metodología de investigación, se incentiva el espíritu investigativo como una forma de apropiación y aprendizaje para la vida. El proyecto se convierte en eje dinamizador para resolver problemas, crear propuestas o alternativas requeridas y valoradas en contextos generales o específicos.

La Práctica Aplicada busca integrar al estudiante en un contexto de aprendizaje en campo real relacionado con el rol a desempeñar como profesional. Se propone posibilitar al estudiante un escenario más para que amplíe, contraste y adquiera conocimientos, información, habilidades y profundice en las Competencias específicas de su ejercicio profesional en el ámbito laboral.

Los Dispositivos, retomando a Bernstein, regulan, delimitan y refuerzan sus realizaciones pedagógicas y a su vez generan interacción, permiten la gestión y dinamizan los procesos de aprendizaje en los estudiantes de manera significativa y autónoma.

El Dispositivo Comunicativo es el eje orientador de motivación, estímulo para el encuentro dialogado en la reflexión, el debate y la propuesta.

El Dispositivo Investigativo es generador de espíritu de búsqueda permanente y disposición a la investigación. Se activa a partir de la reflexión crítica como una habilidad metacognitiva (pensar sobre el pensamiento), habilidad creativa y con postura crítica. Por ello, a través del dispositivo investigativo se busca que los estudiantes piensen en cómo experimentar, cómo interpretar, como construir, incluyendo su pensamiento (saber) sentimientos y nece- 
sidades propias de su contexto. Lo cual requiere que en las prácticas investigativas, los tutores impliquen el pensar y el actuar de manera integrada, buscando que realicen conexiones, interconexiones, múltiples interrelaciones entre varios puntos de vista o posiciones.

El Dispositivo Tecnológico es asumido como dispositivo tecnológico-social, se busca que medien las prácticas de conectividad con las prácticas de producción de conocimiento. Se espera que el estudiante aprenda a trascender el nivel meramente instrumental.

En este sentido, el dispositivo, actúa como sistema de diversas disposiciones cognitivas para que los estudiantes efectúen múltiples acciones en pro de lograr su desempeño a través de un ambiente de aprendizaje mediado.

Por otra parte, permite selección, jerarquía, uso de la información, producción en prácticas de conectividad en la red y en el aula. Adicionalmente, involucra interactividad, relaciones que median la producción, circulación y consumo de diversas prácticas comunicativas. Este dispositivo es considerado y utilizado como eje de sentido en donde la comunidad académica recepta y construye con relación a sus prácticas cotidianas, por ejemplo, el uso de redes sociales. Se busca además que el dispositivo permita resignificar y reelaborar contenidos a partir de la experiencia.

Retomando las características fundamentales de las reglas planteadas por Bernstein, con la regla Distributiva los dispositivos pedagógicos (la comunicación, la investigación y la tecnología) buscan integrarse en el currículo uniendo lo cono- cido por el estudiante (saberes previos) con lo desconocido (nuevos conocimientos, conocimientos disciplinares e interdisciplinares). Como dispositivos pedagógicos generan la brecha discursiva que permite ir de lo impensable a lo posible. Dado que estos dispositivos se accionan en escenarios como el aula virtual, el proyecto y la práctica, en cada uno de estos escenarios se hacen posibles alternativas de nuevas prácticas, distribuyen las acciones pedagógicas determinando roles, condiciones y reglas entre tutores-estudiantes y entre estudiantes.

Con la regla de Recontextualización, los discursos de estos tres dispositivos se accionan, interactúan, relacionan y circulan unos a través de otros. Por ejemplo, la comunicación como discurso, es accionada por la investigación con el apoyo de la tecnología. El discurso de la tecnología es accionado por la investigación y la comunicación. En este sentido se da un engranaje entre Comunicación, Investigación y Tecnología en donde los discursos de cada una de las tres competencias como dispositivos son instruccionales y regulatorios entre sí.

Es necesario resaltar que esta regla desde la perspectiva de Bernstein, construye el discurso pedagógico y el modo en que el discurso instruccional es inserto en el discurso regulativo, lo que significa que como dispositivos pedagógicos cada uno por sí mismo construye su discurso pedagógico y el modo en que el discurso instruccional se inserta en el discurso regulatorio.

Lo instruccional, tal como lo plantea Bernstein, crea habilidades que deben ser adquiridas y sus relaciones entre sí. Lo regulativo dispone las reglas que consti- 
tuyen el orden, la relación y la identidad. Por lo tanto, cualquiera de los dispositivos opera de manera individual y a su vez establecen relaciones, conservando su identidad, a través de discursos, valores, normas y habilidades.

Con la regla Evaluativa, la práctica para el aprendizaje se concibe desde la perspectiva de Miklos (2010) como "una construcción de aprendizajes integrales, integrables y transferibles a la realidad". Por ello, los dispositivos buscan integrar conocimientos-contextos, teorías-practicas, investigación-proyección social, formación dual a la que hace referencia el eje institucional para la Educación Virtual.

Adicionalmente, se refuerza esta regla con la práctica profesional aplicada, por un lado, para posibilitar y confrontar conocimientos, información, habilidades y competencias requeridas en el ejercicio profesional para el desempeño laboral; por otro lado, para evaluar y retroalimentar el currículo, observando y analizando de manera crítica si se están logrando los objetivos propuestos.

\section{Conclusiones}

Las Competencias genéricas, como ejes transversales, dinamizan y gestionan el desarrollo personal y profesional y tienen como propósito el avance y evolución en términos cognitivos durante la carrera. Además de asumir la formación desde el conocimiento (saber), se ocupan del saber hacer, actuar, ser y emprender. Desde esta perspectiva, las Competencias genéricas son una expresión concreta de los diversos recursos que pone en juego un estudiante cuando lleva a cabo una actividad, cuando hace uso o manejo de lo que debe hacer, de lo que sabe de manera integrada, no del conocimiento aislado para dar cuenta de su desempeño. Si bien desde el sentido de las competencias se busca medir el desempeño, para el Politécnico este adquiere sentido cuando prevalecen las actitudes, valores, motivaciones además de conocimientos, habilidades y destrezas.

En lo que atañe a los dispositivos podemos concluir que permiten, a través de la multiplicidad de experiencias del hacer y del actuar cotidiano, una práctica cultural, en donde la comunicación y la interacción a través del diálogo, las actividades, la participación, la investigación, el uso de recursos, la creatividad no solo se dinamizan sino que se convierten en una capacidad de transformar. Los dispositivos, a través de los diferentes ambientes de aprendizaje, permiten compartir saberes, conocimientos, experiencias, acceso y manejo de la tecnología, innovaciones, investigaciones, resolución de problemas y conflictos, así como satisfacer necesidades. Por otra parte, los dispositivos pedagógicos posibilitan adquirir nuevos lenguajes y a su vez entender la vida cotidiana.

\section{Bibliografía}

1. Bernstein, B. (1998), Pedagogía control simbólico e identidad: teoría investigación y crítica, España, Editorial Morata.

2. Bernstein, B. (PDF sin fecha) Dispositivo Pedagógico, Reglas Constitutivas, página http://www.uneduc.cl/educacion/6667_bernstein.pdf recuperado en agosto de 2010 (pág. 2,3 y 7). 
3. Miklos, T. (2009) Visiones competentes sobre competencias ( aproximaciones pedagógicas ), Red de Revistas Científicas de América Latina y el Caribe, España y Portugal Sistema de Información Científica Redalyc, Revista del centro de investigaciones, Universidad La Salle, Vol. 8, n.0 32 , julio-dic. 2009, pp. 5-25 México.

4. Ministerio de Educación Nacional, MEN, (2009), Competencias genéricas en Educación Superior. Boletín Informativo n.0 13 Diciembre-2009, Bogotá, Panamericana Formas e impresos, S.A.

5. Politécnico Grancolombiano. Filosofía Institucional. Misión. Bogotá, documento publicado en la página http://www.poligran.edu.co/eContent/newsdetail.asp?id $=276 \&$ idcompany $=28 \&$ idscategory $=65 \&$ itemmenu $=2 \_250 \#$, consultado junio 2010.

6. Politécnico Grancolombiano. (2004). Proyecto Educativo Institucional PEI, Bogotá, (pág. 8), documento publicado en la página http://www.poligran.edu.co/ comunica/documentosportal/pei.pdf, consultado junio 2010.

7. Politécnico Grancolombiano. (2008a). Documento Interno de trabajo Institucional. Planeación Estratégica 2008- 2013. Bogotá.

8. Politécnico Grancolombiano. (2009). Modelo Institucional, Bogotá, documento interno.

9. Politécnico Grancolombiano. (2009). Modelo Comunicativo. Bogotá, documento interno.

10.Politécnico Grancolombiano. (2008b). Investigación Formativa. Bogotá, documento interno, Departamento de Investigación.

11. Torrado, M. (2000), Educar para el desarrollo de las competencias: una propuesta para reflexionar. Resumido por la profesora Swapna Puni Estévez Singh, bachiller en ciencias, del libro COMPETENCIAS Y PROYECTOS PEDAGOGICOS Universidad Nacional de Colombia Bogotá, página: http://panelsd.iespana.es/ col00020.htm., recuperado: agosto de 2010.

\section{Consultas en línea}

1. Politécnico Grancolombiano. Filosofía Institucional. Misión. Bogotá, documento publicado en la página http://www.poligran.edu.co/eContent/newsdetail.asp?id $=276 \&$ idcompany $=28 \&$ idscategory $=65 \&$ itemmenu $=2 \_250 \#$, consultado junio 2010.

2. Politécnico Grancolombiano. Proyecto Educativo Institucional PEI, Bogotá, 2004 (pág. 8), documento publicado en la página http://www.poligran.edu.co/ comunica/documentosportal/pei.pdf, consultado junio 2010.

3. Torrado, M. Educar para el desarrollo de las competencias: una propuesta para reflexionar. Resumido por la profesora Swapna Puni Estévez Singh, bachiller en ciencias, del libro Competencias y proyectos pedagógicos - Universidad Nacional de Colombia Bogotá, 2000. página: http://panelsd.iespana.es/col00020.htm., recuperado: agosto de 2010. 